\title{
Unmanned Aerial Vehicle (UVA) Bionic Wing Design and Performance Analysis
}

\author{
Hua Xin ${ }^{1, \text { a }}$, QinShuo ${ }^{1, b}$ KaiLuo $^{1, c}$ weihongliang ${ }^{2, c}$ \\ ${ }^{1}$ The air force air university, CHINA \\ ${ }^{2}$ Changchun power supply company ,CHINA \\ ahuaxin0719@163.com, ’qinshuo@163.com, 'luokao@163.com
}

\begin{abstract}
Keywords: For the rest of the Bionic; Unmanned aerial vehicle (uav); The aerodynamic performance; The numerical simulation
\end{abstract}

\begin{abstract}
Key problems in view of the urgent long-endurance uav, the research of the application of bionics to no one wing design. With the wings of a certain unmanned aerial vehicle as the research object, is good at gliding gull for bionic prototype. Design imitation airfoil seagull flat wings and wing tip winglet two kinds of bionic wing. For bionic wings with conventional using CFD simulation method is used to numerically simulate the wing, the study found that uavs using bionic wings can effectively improve the flow field distribution of the wing, decrease on the wing surface flow separation, and reduce the aerodynamic drag coefficient, thus improve the wing lift-to-drag ratio. The results show that two kinds of bionic wings than standard average lift-to-drag ratio increased by $16.5 \%$ and $17.6 \%$, respectively. In this paper the simulation results provide certain reference for the design of the uav wing.
\end{abstract}

\section{Introduction}

UAV wing aerodynamic characteristics has important influence on the performance of the unmanned aerial vehicle (uav), near space, on the one hand, due to the thin air, flying low operation pressure, low Reynolds number (millions of magnitude), to meet the requirements of unmanned aerial vehicle (uav) big load, must improve the lift. Because long-endurance flight, on the other hand, must reduce flight resistance to reduce fuel consumption. So the wing drag reduction rising of high-altitude long-endurance uav design more and more important.

Bionics is a comprehensive discipline, from the date of birth, showing strong vitality. Combining application in engineering bionics content is rich and colorful, with the measuring method of bionics continue to improve, not only development of biology, and also make the bionics to channel carrying their precious materials to various industries and rich nutrition. Seagull is a kind of good at gliding birds, as long as there is a little bit of updraft can glide for a long time on the sea, this is beneficial to save energy in the long flight. Based on bionics theory, selects the gull gliding wings airfoils, when applied to the uav wing design, design a straight wing, the numerical simulation. Through the comparison with conventional wings, to analyze the flow field characteristics and drag reduction mechanism of rising, for near space unmanned aerial vehicle (uav) give some reference to the design of the wing.

\section{Wing model establishment and meshing}

Section This article refer to a certain type of flying drones aspect ratio, based on the bionic airfoil show to stretch and become, airplane wing root chord length $C=216 \mathrm{~mm}$, the wing tip chord length $\mathrm{C}=108 \mathrm{~mm}$, half a wingspan of $962 \mathrm{~mm}$ long. Conventional wing and bionic wing model as shown in figure 1. Structured grid was taken to the discrete simulation computation domain according to the wing shape characteristic, computational domain front end of the wing leading edge is 12.5 times the chord length, from the back-end is apart from the trailing edge 20 times the chord length, wing tips and its corresponding face is 15 times the chord length. Grid computing domain as shown in figure 2. Grid generation for thinning near the edge of the wing, the wing 
around encryption processing is shown in figure 3. The grid number about 1.1 million.

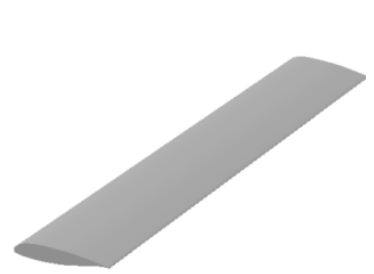

Fig.1 wing model

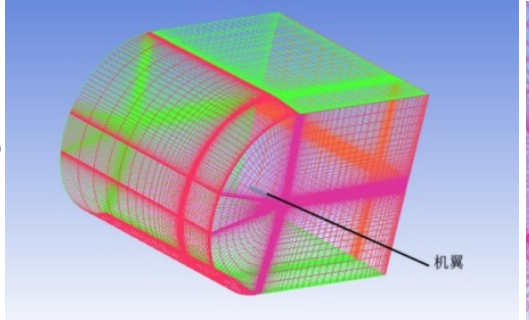

Fig. 2 meshing calculation domain Fig. 3 wing surface grid

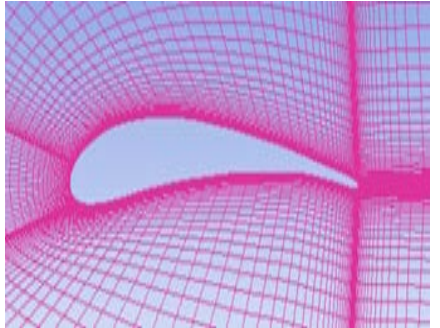

\section{Fluid calculation model}

For $\mathrm{N}$ - S equation, continuous equation, momentum equation and energy equation of general form can be written as the following:

$$
\frac{\partial(\rho \phi)}{\partial t}+\operatorname{div}(\rho U \phi)=\operatorname{div}(\Gamma \operatorname{grad} \phi)+S
$$

This article selects the S - A turbulence model for numerical simulation. S - A turbulence model is an equation model, different from other single equation model, this model is directly based on experience and dimensional analysis, starting from the simple flow, the final control equation is obtained directly. Therefore its ability to handle complex flow, fault-tolerant performance is good, suitable for simulation of the internal flow and moderately complex outflow and pressure gradient of the boundary layer flow. For encryption the boundary layer of the grid, adopt the S - A turbulence model is easier to capture the high field the wing of the flow separation, therefore in the numerical calculation, the $\mathrm{S}$ - A model for this paper. Model of single equation is variable.

$$
\rho \frac{d \tilde{v}}{d t}=G_{v}+\frac{1}{\sigma_{\tilde{v}}}\left[\frac{\partial}{\partial x_{j}}\left\{(\mu+\rho \widetilde{v}) \frac{\partial \tilde{v}}{\partial x_{j}}\right\}+C_{b 2}\left(\frac{\partial \tilde{v}}{\partial x_{j}}\right)\right]-Y_{V}
$$

This article selects $20 \mathrm{~km}$ altitude stratosphere environment for computing environment, temperature $216.5 \mathrm{~K}$, pressure 5460 pa density of 0.08991 . In the process of numerical simulation using the coupled solver (coupled), choosing implicit format, equation using the Gauss - Seidel method with AMG method. Various boundary conditions are set as follows, entry and exit of computational domain are far field boundary selection pressure, flow Mach number is set to 0.3, 0.7 respectively, select to flow Angle of attack and respectively; Set to a fixed wing wall; And connected to the wing of the boundary is set for the symmetry plane.

\section{Results analysis}

In conventional wing and bionic wing are simulated calculation as shown in figure 4. Can be seen from the diagram, the bionic wing with conventional airfoil drag coefficient and lift-to-drag ratio coefficient increases with the increase of Angle of attack. Can be seen from the figure 4 when $\mathrm{Ma}=0.4$, the drag coefficient in Angle of attack for the resistance coefficient of minimum 3 degrees. Lift-to-drag ratio in $\mathrm{Ma}=0.7$ lift-to-drag ratio standard wings increased by $10.3 \%$. Fig.5 for $\mathrm{Ma}=0.6$, streamline distribution on the surface of the wing, there are obvious at the rear of the conventional wing flow separation, bionic trailing edge separation was not significant, indicating that the bionic wing can effectively improve the flow field, delay flow separation and increase the lift.

\section{The bionic wings optimization}

Can see from the above analysis of the bionic wing wing aerodynamic performance is superior to the standard, but can be seen from the flow chart 5 (b) air extending to flow more apparent, cause more apparent flow separation. Thus will design the wing tip winglet bionic wings. As shown in 
fig.6
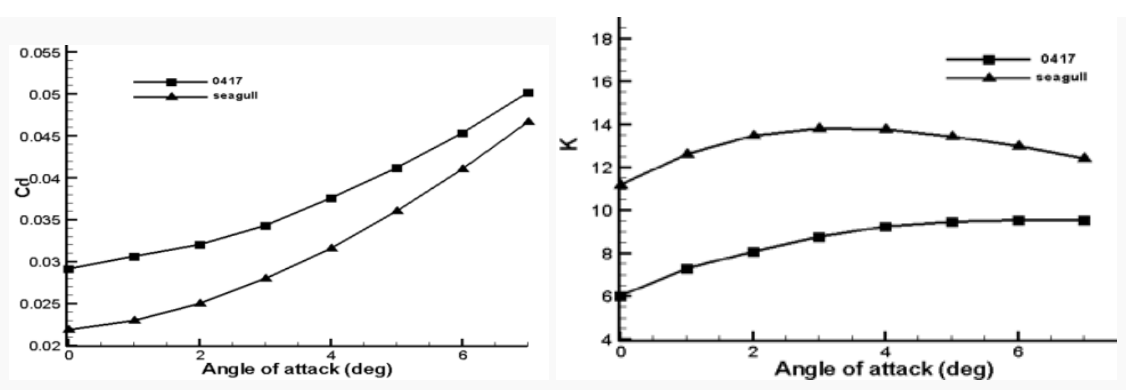

Fig. 4 bionic wings with conventional lift, drag coefficient and lift-to-drag ratio change curve

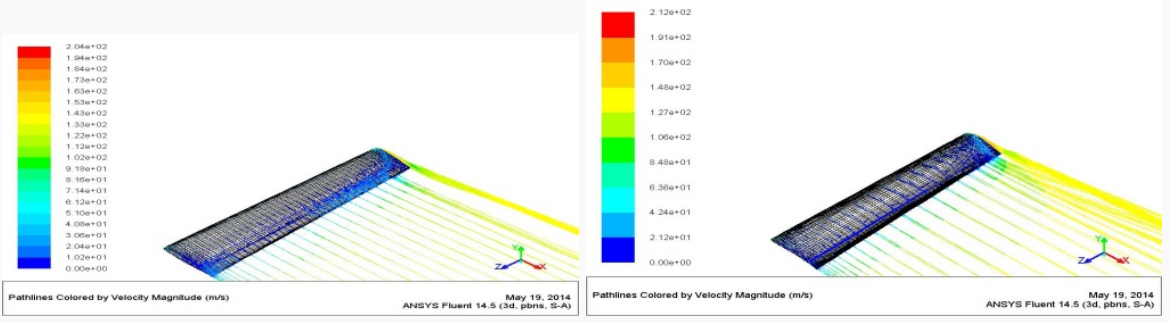

Fig. $5 \mathrm{Ma}=0.6, \quad \alpha=3^{\circ}$ The wing surface flow chart
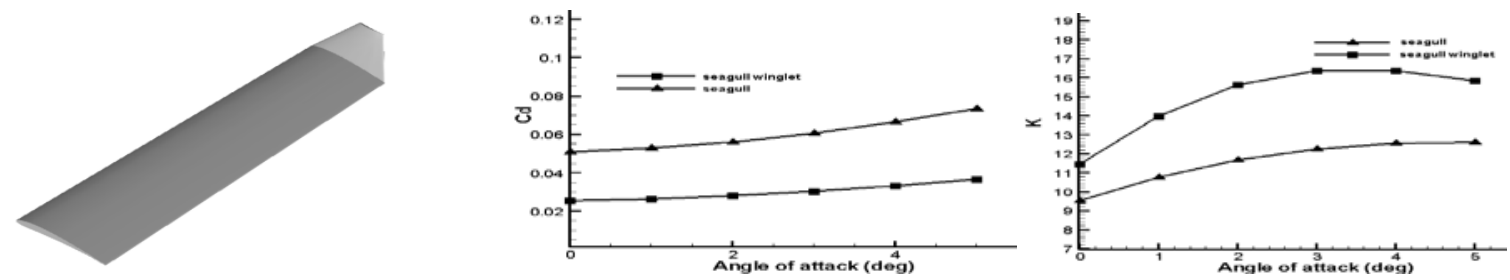

Fig. 6 plus wing tip winglet model of bionic wings

Fig. 7 with winglet and bionic wings straight airfoil drag and the lift-to-drag ratio change curve

\section{Results analysis}

Can be seen from the diagram, equipped with winglet bionic wing at Angle of attack for the reach maximum lift-to-drag ratio is 15.83, relatively flat bionic wings in Angle of attack for reach maximum lift-to-drag ratio of 12.61, up 20.13\%. Under 0.4 Ma with wing tip winglet bionic airfoils with straight average bionic airfoil lift-to-drag ratio of $14.94 \%, 11.56 \%$ respectively. Fig. 8 is flat wings and add wing tip winglet near the wing tip section stress nephogram, wing lift is produced by the pressure difference between the top and bottom surface. As can be seen when $\mathrm{z}=0.9 \mathrm{~m}$, negative pressure zone is mainly located at the side of the wings on the first part is mainly characterized by suction, suction lift for major contributor. When $\mathrm{z}$ increases, that is, the closer the wing tips, negative pressure zone area gradually decreases and move back, causing a significant decrease the lift and at the same time the suction force component in horizontal direction and wing movement in the opposite direction, this will produce larger pressure drag. After add wing tip winglet, effectively improve the air condition, pressure distribution change is not severe.

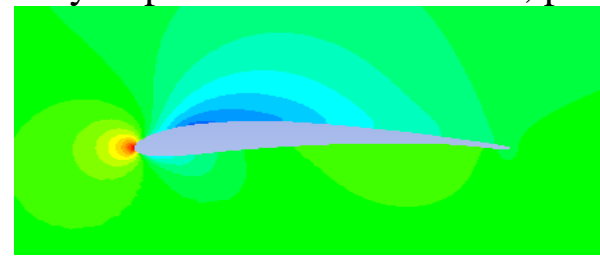

(a) $\mathrm{z}=0.9 \mathrm{~m}$

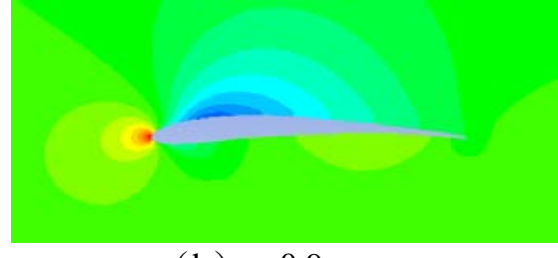

(b) $\mathrm{z}=0.9 \mathrm{~m}$ 


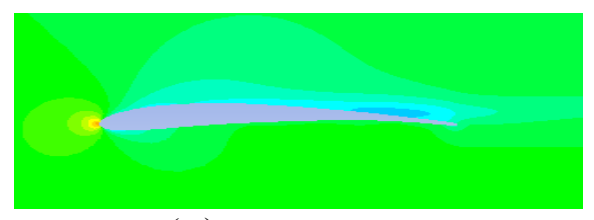

(c) $\mathrm{z}=0.95 \mathrm{~m}$

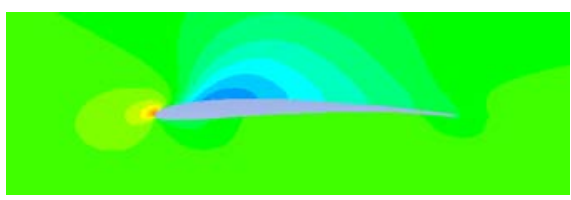

(d) $\mathrm{z}=0.95 \mathrm{~m}$

Fig. 8 wings near the wing tip section stress nephogram

Can be seen from the figure 8 (b), because the upper and lower surface pressure difference causes air currents to exhibition will happen to the movement, formed in the tops of wing trailing edge wingtip vortex. Because of the existence of wingtip vortex, within the scope of the entire wingspan will induce a downwash velocity, downwash velocity cause changes in the actual lift direction, effective lift decreases, and at the same time, the actual lift component for induced resistance and resistance in the same direction. In fig.8(b), the wings of wingtip vortex is very obvious, airflow downwash effect, formation of induced resistance may also be larger. Bionic wings, after adding wing tip winglet on the surface of the wing has improved streamline.

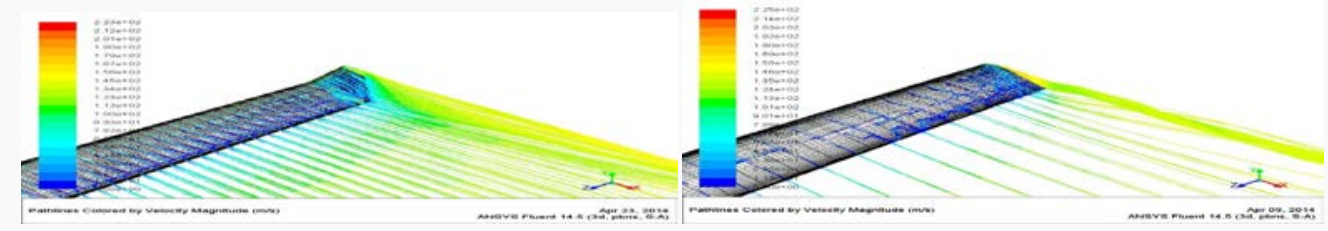

Fig. $9 \mathrm{Ma}=0.6$ and add wing tip winglet bionic wings straight wing surface flow chart

as shown in figure 7 (a). Because of the existence of the wing tip winglet, cut off the wings up and down the flow around, makes the flow surface of the wing of the exhibition to slow down and up and down the wing surface more smooth streamline, delayed the wing tips in air separation in advance. With time to carefully sharp trailing edge wingtip vortex is abate, downwash Angle and downwash velocity is reduced, so that the induced resistance decreases.

\section{Conclusion}

This paper, by means of numerical simulation, compares the bionic wing airfoil with conventional airfoil aerodynamic characteristics of the wings in the sky, it is concluded that the pressure distribution, flow field distribution on the surface of the wings, and analyzes the bionic wing drag reduction principle of rising. The study found that the bionic wing can effectively reduce the pressure drag, increase the wings on the surface of the upper limb negative pressure area, so as to achieve the aim of reducing resistance, increase the wing lift. But can be seen from the flow chart of bionic wings, bionic wings eyes show to flow is more obvious, thus optimize the bionic wing with a wing tip winglet, compared by the method of numerical simulation with winglet bionic wing airfoil and straight after the bionic wings near the aerodynamic characteristics of the space, analyzes the mechanism of drag reduction add up. Calculation results show that after adding wing tip winglet, imitation of life carefully tip vortex wingtip effect is abate, reduces the downwash velocity slows the wing surface flow separation, thus achieved the effect of drag reduction add up.

\section{References}

[1] Hua Xin. Numerical Simulation And Aerodynamic Performance Comparison Between Seagull Aerofoil and NACA 4412 Aerofoil under Low-Reynolds[J]. Advances in Natural Science, 2010,3 (2) : 244-250.

[2] Hua Xin. High Altitide and Long Endurance Unmaned Aerial Vehicle Bionic Airfoil Research [3] Y. Mishing, in: Diffusion Processes in Advanced Technological Materials, edtied by D. Gupta

[3] Hua Xin. Base on imitation seagullairfoil UVA wing numerical simulation[J]2012,3(1)323-325. 